\title{
COMUNICAÇÃ̃O
}

\section{INFLUÊNCIA DA ÁREA DE AMOSTRAGEM NA DETERMINAÇÃO DE PERDAS TOTAIS NA COLHEITA DE SOJA}

\author{
Influence of the area of sampling of the frames in the determination of total losses \\ of soybean harvesting
}

\author{
Felipe Thomaz da Câmara', Rouverson Pereira da Silva², Afonso Lopes², Carlos Eduardo Angeli Furlani \\ Danilo César Cecchio Grotta ${ }^{1}$, Gustavo Naves dos Reis ${ }^{1}$
}

\begin{abstract}
RESUMO
A metodologia, atualmente utilizada, para a determinação das perdas na colheita de soja, que consiste na utilização de armações de $2 \mathrm{~m}^{2}$, vem fornecendo resultados com alto coeficiente de variação, o que dificulta detectar diferenças significativas entre os tratamentos, mesmo que esta diferença apresente valores numéricos muito altos. Em função deste problema, objetivou-se avaliar o efeito das armações sobre a quantificação das perdas totais na colheita da cultura da soja, alterando-se a velocidade de deslocamento da colhedora e a abertura entre o cilindro e o côncavo. O delineamento experimental foi em blocos casualizados, em esquema fatorial $2 \times 3 \times 2$, totalizando 12 tratamentos com quatro repetições. Os resultados evidenciaram que o coeficiente de variação para a armação de $2 \mathrm{~m}^{2}$ foi de $88,26 \%$, enquanto que para a armação de $3 \mathrm{~m}^{2}$ este valor foi de $32,85 \%$, e que com a armação de $2 \mathrm{~m}^{2}$ foram detectadas perdas totais de $45 \mathrm{~kg} \mathrm{ha}^{-1}$, enquanto que com a de $3 \mathrm{~m}^{2}$ quantificou-se $73 \mathrm{~kg} \mathrm{ha}^{-1}$, indicando que a área das armações interfere na quantificação das perdas totais na cultura da soja.
\end{abstract}

Termos para indexação: Colheita mecanizada, velocidade de deslocamento, abertura do côncavo.

\section{ABSTRACT}

The currently methodology used for the losses determination in the soybean harvest, that consists of the use of frames of $2 \mathrm{~m}^{2}$, comes supplying resulted with high coefficient of variation, what it makes it difficult to detect significant differences between the treatments, exactly when this difference arrives to be almost the double of one for the other. In function of the related one above, this work had for objective to evaluate the effect of the frames on the quantification of the total losses in the harvest of the culture of the soybean (Glycine $\max (\mathrm{L}$.) Merrill), getting excited it speed of displacement of the harvester and the opening between the cylinder and the concaves. The used experimental design was randomized blocks, in factorial scheme $2 \times 3 \times 2$, totaling 12 treatments with four repetitions. The results evidenced that the variation coefficient for the frame of $2 \mathrm{~m}^{2}$ was of $88.26 \%$, while for the frame of $3 \mathrm{~m}^{2}$ this value was of $32.85 \%$, and that with the frame of $2 \mathrm{~m}^{2}$ total losses of $45 \mathrm{~kg} \mathrm{ha}^{-1}$ were detected, while with the one of $3 \mathrm{~m}^{2} 73 \mathrm{~kg} \mathrm{ha}^{-1} \mathrm{was}$ quantified, it differentiates this significant one to $1 \%$ of probability for the Teste $\mathrm{F}$, indicating that the area of the frames interferes in the quantification of the total losses in the culture of the soy.

Index terms: Mechanical harvesting, displacement speed, opening of the concave.

(Recebido em 21 de dezembro de 2005 e aprovado em 22 de janeiro de 2007)

A colheita é a última etapa de campo do processo produtivo e, segundo Moraes et al. (1996), este é um fato suficiente para justificar um estudo do assunto, pois qualquer fator que venha interferir de forma negativa nesta etapa, como por exemplo as regulagens da máquina, pode pôr em risco a rentabilidade do produtor e neste contexto, as perdas na colheita são de vital importância.

As más regulagens da colhedora ocorrem, na maioria das vezes, pela falta de operadores capacitados para tal função, como constatado por Silva et al. (1998). Dentre as regulagens para obtenção de uma colheita eficiente, com níveis de perdas dentro do limite tolerável, que para a cultura da soja é de $1 \mathrm{sc} \mathrm{ha}^{-1}\left(60 \mathrm{~kg} \mathrm{ha}^{-1}\right)$, destacam-se a velocidade do molinete, a velocidade de operação e as regulagens do sistema de trilha e de limpeza (EMBRAPA, 2003). A falta de monitoramento das perdas, juntamente com a ausência de metodologias adequadas para estimálas, têm contribuído para elevados níveis de perdas.

\footnotetext{
'Engenheiro Agrônomo, Doutorando em Agronomia - Departamento de Engenharia Rural/DER - Universidade Estadual Paulista Júlio de Mesquita Filho/UNESP - Via de Acesso Professor Paulo Donato Castellane, Km 5 - 14890-044 - Jaboticabal, SP - felipetdacamara@yahoo.com.br; dcgrotta@zipmail.com.br; gnrunesp@hotmail.com

${ }^{2}$ Engenheiro Agrícola, Professor, Doutor em Agronomia - Departamento de Engenharia Rural/DER - Universidade Estadual Paulista Júlio de Mesquita Filho/UNESP - Via de Acesso Professor Paulo Donato Castellane, Km 5 - 14890-044 - Jaboticabal, SP - Bolsista de Produtividade em Pesquisa - CNPq - rouverson@fcav.unesp.br; afonso@fcav.unesp.br

${ }^{3}$ Engenheiro Agrônomo, Professor Adjunto - Departamento de Engenharia Rural/DER - Universidade Estadual Paulista Júlio de Mesquita Filho/ UNESP - Via de Acesso Professor Paulo Donato Castellane, Km 5 - 14890-044 - Jaboticabal, SP - Bolsista de Produtividade em Pesquisa -CNPq furlani@fcav.unesp.br
} 
Existem diversas variações na metodologia empregada para este fim, porém a principal modificação entre elas é a área da armação utilizada para quantificar as perdas. De acordo com Costa \& Tavares (1995), a área da armação é variável com o tamanho da plataforma de corte da colhedora, sendo esta área delimitada por dois pedaços de madeira de $0,50 \mathrm{~m}$, unidas por fios de barbante nas duas extremidades, com comprimento igual à largura da plataforma de corte. Assim a área das armações utilizadas varia de acordo com o tamanho da plataforma.

Por outro lado, Portella (2000a) define que a área da armação deve ser de $1 \mathrm{~m}^{2}$, com o tamanho dos fios de nylon igual ao comprimento da plataforma de corte da colhedora e os pedaços de madeira de comprimento variável, determinado pelo quociente da área da armação $\left(1 \mathrm{~m}^{2}\right)$ pelo comprimento da plataforma de corte da colhedora.

Entretanto, a metodologia mais empregada atualmente é a sugerida por Mesquita et al. (1998), que consiste em definir a área para a armação de $1 \mathrm{~m}^{2}$ para arroz, e de $2 \mathrm{~m}^{2}$ para milho e soja, com a confecção das armações semelhante ao sugerido por Portella (2000a).

Mesquita \& Gaudêncio (1982) desenvolveram um copo medidor, sendo inicialmente recomendado a área de amostragem idêntica ao método descrito por Costa \& Tavares (1995), e posteriormente $2 \mathrm{~m}^{2}$, onde todos os grãos soltos e nas vagens são coletados e colocados dentro de um copo graduado, permitindo a leitura das perdas diretamente em sc $\mathrm{ha}^{-1}$, na coluna correspondente a cada cultura.

Porém, ao serem empregadas estas metodologias em trabalhos científicos observam-se altos coeficientes de variação nos experimentos, o que prejudica a análise estatística dos mesmos, uma vez que diferenças numéricas relativamente grandes entre tratamentos, são estatisticamente iguais, como foi observado por vários autores trabalhando com soja, dentre os quais pode-se citar Carvalho Filho et al. (2004), Costa et al. (2002), Mesquita et al. $(1999,2001,2002)$ e Pinheiro Neto \& Gamero (1999). Segundo Campos et al. (2005), os altos valores encontrados para o coeficiente de variação, são justificados pela alta variabilidade da amostra.

Por outro lado, provavelmente em função do alto coeficiente de variação, vários autores trabalhando com diferentes culturas, tais como Hamer \& Peske (1997), Portella (2000b) e Silva et al. (2004) optaram por não realizar a análise estatística, apresentando os resultados por meio de curvas de regressão.

Objetivou-se avaliar dois tamanhos de armações utilizadas para medição das perdas totais na colheita mecanizada da soja, em função da velocidade de deslocamento e da abertura entre o cilindro de trilha e o côncavo, verificando o comportamento dos coeficientes de variação para cada armação utilizada.

$\mathrm{O}$ experimento foi conduzido em área do Departamento de Engenharia Rural da Universidade Estadual Paulista, Campus de Jaboticabal-SP, localizado na Latitude $21^{\circ} 14^{\prime} 28^{\prime \prime}$ S e Longitude $48^{\circ} 17^{\prime} 12^{\prime \prime}$ W. Aárea apresenta altitude média de $559 \mathrm{~m}$, declividade média de $4 \%$ e clima Cwa (subtropical), de acordo com a classificação de Köeppen. O solo da área experimental foi classificado como Latossolo Vermelho Eutroférrico típico, relevo suave ondulado por Andrioli \& Centurion (1999), conforme o Sistema Brasileiro de Classificação de Solos (EMBRAPA, 1999).

A cultivar de soja colhido foi Conquista, com produtividade média de $1100 \mathrm{~kg} \mathrm{ha}^{-1}$, e no momento da colheita o mesmo apresentava teor de água de $9 \%$, com a finalidade de permitir uma melhor avaliação da interferência do tamanho da armação na quantificação das perdas totais na cultura da soja.

Foi utilizada colhedora SLC 1165, com potência de $103 \mathrm{~kW}(140 \mathrm{cv})$ e plataforma de 3,80 metros, trabalhando com rotação do molinete de $30 \mathrm{rpm}$ e rotação do cilindro de trilha de $550 \mathrm{rpm}$.

Foram utilizadas duas armações no experimento (2 $\mathrm{m}^{2}$ e $3 \mathrm{~m}^{2}$ ), confeccionadas com dois pedaços de madeira, com $0,53 \mathrm{~m}$ de comprimento para a armação de $2 \mathrm{~m}^{2}$ e de $0,79 \mathrm{~m}$ para a armação de $3 \mathrm{~m}^{2}$, sendo os mesmos unidos nas duas extremidades por fios de nylon com comprimento igual à largura da plataforma de corte $(3,80 \mathrm{~m})$.

Cada parcela experimental ocupou área de $114 \mathrm{~m}^{2}$, com $30 \mathrm{~m}$ de comprimento por $3,80 \mathrm{~m}$ de largura, referente à largura da plataforma de corte da colhedora, sendo reservado intervalos de $15 \mathrm{~m}$ entre as parcelas, cuja finalidade foi permitir as alterações das regulagens da colhedora para a próxima parcela. As perdas totais foram obtidas colocando-se as armações no solo, logo após a passagem da colhedora, coletando-se os grãos presentes na área de amostragem, que posteriormente foram pesados e transformados para $\mathrm{kg} \mathrm{ha}^{-1}$.

$\mathrm{O}$ delineamento utilizado foi em blocos casualizados, em esquema fatorial $2 \times 3 \times 2$, com 12 tratamentos e quatro repetições, totalizando 48 observações.

As combinações dos tratamentos foram: dois tamanhos de armações $\left(\mathrm{A}_{1}=2 \mathrm{~m}^{2}\right.$ e $\left.\mathrm{A}_{2}=3 \mathrm{~m}^{2}\right)$, três velocidades de deslocamento da colhedora $\left(\mathrm{V}_{1}=3 \mathrm{~km} \mathrm{~h}^{-1}\right.$, $\left.\mathrm{V}_{2}=4 \mathrm{~km} \mathrm{~h}^{-1} \mathrm{e} \mathrm{V}_{3}=6 \mathrm{~km} \mathrm{~h}^{-1}\right)$ e duas aberturas entre $\mathrm{o}$ cilindro e o côncavo $\left(\mathrm{Ac}_{1}=40 \mathrm{~mm}\right.$ e $\left.\mathrm{Ac}_{2}=29 \mathrm{~mm}\right)$, mantendose as demais regulagens da colhedora constantes. 
Os dados obtidos foram tabulados e submetidos à análise de variância (Teste F) e ao teste de comparação de médias de Tukey a 5\% de probabilidade, utilizando-se o programa computacional Estat.

Na Tabela 1 são apresentados os resultados obtidos com as duas armações, com relação às perdas totais na colheita da cultura da soja, variando-se a velocidade de deslocamento e a abertura entre o cilindro e o côncavo, onde cada valor representa a síntese de duas observações para velocidade e três observações para a abertura do côncavo.

Verifica-se que, para a armação de $2 \mathrm{~m}^{2}$, não foi observada diferença significativa para o fator velocidade de deslocamento da colhedora (V), mesmo com a velocidade $\mathrm{V}_{2}$ apresentando valores de perdas $94 \%$ maiores que a $\mathrm{V}_{3}$. Este resultado demonstra que a metodologia para determinação de perdas na colheita deve ser revista, pois a semelhança estatística encontrada não reflete a realidade devido ao alto coeficiente de variação (CV\%) encontrado $(88,26 \%)$, valor este semelhante aos encontrados por outros autores como Kunz et al. (2005) com 66,3\%, Mesquita et al. (2002) com 64,8\%, Mesquita et al. (2001) com 69,6\%, $66,9 \%$ e $71,7 \%$ e Mesquita et al. (1999) com 69,2\%. O alto valor de CV\% e a diferença mínima significativa (DMS) de $51,2 \mathrm{~kg} \mathrm{ha}^{-1}$, prejudicaram a análise dos resultados, uma vez que não se pode concluir que $\mathrm{V}_{2}$ ocasiona maiores perdas totais do que $\mathrm{V}_{3}$, mesmo provocando perdas totais 94\% maiores. Quando se utilizou a armação de $3 \mathrm{~m}^{2}$, também não houve diferença estatística significativa, porém $\mathrm{V}_{1}$ foi apenas $21,5 \%$ maior do que $\mathrm{V}_{3}$, com $\mathrm{CV} \%$ de $32,85 \%$ e DMS de 30,94 $\mathrm{kg} \mathrm{ha}^{-1}$, valores estes bem menores do que os obtidos com a armação de $2 \mathrm{~m}^{2}$, o que permite avaliar com maior confiabilidade que as velocidades de deslocamento da colhedora não influenciaram nas perdas totais da cultura da soja.

Como pode ser observado na Tabela 1, para o fator abertura entre o cilindro e o côncavo (Ac), não houve diferença estatística significativa para as armações estudadas, e a $\mathrm{Ac}_{2}$ ocasionou perdas totais $17 \%$ maiores do que $A c_{1}$, para a armação de $2 \mathrm{~m}^{2}$, enquanto que para a

TABELA 1 - Síntese da análise de variância nas armações de 2 e 3 metros quadrados para perdas totais na cultura da soja.

\begin{tabular}{|c|c|c|}
\hline \multirow{2}{*}{ Fatores } & \multicolumn{2}{|c|}{ Perdas $\left(\mathrm{kg} \mathrm{ha}^{-1}\right)$} \\
\hline & $A_{1}\left(2 \mathrm{~m}^{2}\right)$ & $A_{2}\left(3 \mathrm{~m}^{2}\right)$ \\
\hline \multicolumn{3}{|l|}{ Velocidade (V) } \\
\hline $\mathrm{V}_{1}$ & $37 \mathrm{a}$ & $79 a$ \\
\hline $\mathrm{V}_{2}$ & $64 \mathrm{a}$ & $75 \mathrm{a}$ \\
\hline $\mathrm{V}_{3}$ & 33 a & $65 \mathrm{a}$ \\
\hline \multicolumn{3}{|c|}{ Abertura do côncavo (Ac) } \\
\hline $\mathrm{Ac}_{1}$ & $41 \mathrm{a}$ & $70 \mathrm{a}$ \\
\hline $\mathrm{Ac}_{2}$ & $48 \mathrm{a}$ & $77 \mathrm{a}$ \\
\hline \multicolumn{3}{|l|}{ Teste F } \\
\hline $\mathrm{V}$ & $1,3996^{\mathrm{NS}}$ & $0,7355^{\mathrm{NS}}$ \\
\hline Ac & $0,2120^{\mathrm{NS}}$ & $0,5545^{\mathrm{NS}}$ \\
\hline VxAc & $0,7936^{\mathrm{NS}}$ & $1,2864^{\mathrm{NS}}$ \\
\hline Media Geral & 45 & 73 \\
\hline DMS (V) & 51,20 & 30,94 \\
\hline DMS (Ac) & 34,28 & 20,72 \\
\hline Desvio Padrão & 39 & 24 \\
\hline C.V.\% & 88,26 & 32,85 \\
\hline
\end{tabular}

Em cada coluna, para cada fator, médias seguidas de mesma letra minúscula não diferem entre si, pelo teste de Tukey a $5 \%$ de probabilidade; ${ }^{\text {NS}}$ : não significativo ( $\left.>>0,05\right)$; dms: diferença mínima significativa; C.V.\%: coeficiente de variação. 
armação de $3 \mathrm{~m}^{2}$, esta diferença foi de apenas $10 \%$. Com relação à DMS, a da armação de $2 \mathrm{~m}^{2}$ foi de $34,28 \mathrm{~kg} \mathrm{ha}^{-1}$, valor este superior ao encontrado para a armação de $3 \mathrm{~m}^{2}$ $\left(20,72 \mathrm{~kg} \mathrm{ha}^{-1}\right)$, permitindo uma análise melhor fundamentada, uma vez que a influência de fatores não controlados foram menores para a armação de $3 \mathrm{~m}^{2}$. Na Tabela 1 pode-se observar também que não houve interação significativa entre a velocidade de deslocamento da colhedora e a abertura entre o côncavo e o cilindro para as duas armações em estudo.

Na Tabela 2 são apresentados os resultados das perdas totais na colheita da cultura da soja, variando-se a área da armação, velocidade de deslocamento e a abertura entre o cilindro e côncavo, onde cada valor representa a síntese de 6 observações para as armações, 4 observações

TABELA 2 - Síntese da análise de variância e do teste de médias, considerando-se as armações como um terceiro fator, para as perdas totais na colheita da soja em kg ha-1.

\begin{tabular}{lc}
\hline Fatores & $\begin{array}{c}\text { Perdas Totais } \\
\mathbf{k g ~ h a}^{-1}\end{array}$ \\
\hline Armações (A) & $45 \mathrm{~b}$ \\
$\mathrm{~A}_{1}$ & $73 \mathrm{a}$ \\
$\mathrm{A}_{2}$ & \\
\hline Velocidade (V) & $58 \mathrm{a}$ \\
$\mathrm{V}_{1}$ & $69 \mathrm{a}$ \\
$\mathrm{V}_{2}$ & $49 \mathrm{a}$ \\
$\mathrm{V}_{3}$ &
\end{tabular}

\begin{tabular}{lc}
\hline Abertura do côncavo (Ac) & $55 \mathrm{a}$ \\
$\mathrm{Ac}_{1}$ & $63 \mathrm{a}$ \\
$\mathrm{Ac}_{2}$ & \\
\hline Teste F & $7,7822^{* *}$ \\
$\mathrm{~A}$ & $1,2895^{\mathrm{NS}}$ \\
$\mathrm{V}$ & $0,5152^{\mathrm{NS}}$ \\
$\mathrm{Ac}$ & $0,7843^{\mathrm{NS}}$ \\
$\mathrm{AxV}$ & $0,0001^{\mathrm{NS}}$ \\
$\mathrm{AxAc}$ & $1,5041^{\mathrm{NS}}$ \\
$\mathrm{VxAc}$ & $0,0665^{\mathrm{NS}}$ \\
$\mathrm{AxVxAc}$ & 60,03 \\
\hline C.V.\% & \\
\hline
\end{tabular}

Em cada coluna, para cada fator, médias seguidas de mesma letra minúscula não diferem entre si, pelo teste de Tukey a 5\% de probabilidade; ${ }^{\text {Ns: }}$ não significativo $(\mathrm{p}>0,05)$; ${ }^{* *}$ : significativo $(\mathrm{p}<0,01)$; c.v.\%: coeficiente de variação. para velocidade e 6 observações para a abertura do côncavo.

Observa-se na Tabela 2 que houve diferença para o fator área da armação, em relação às perdas totais na colheita da soja, com a armação de $3 \mathrm{~m}^{2}$ quantificando perdas $62 \%$ maiores do que a armação de $2 \mathrm{~m}^{2}$. Este resultado indica que a metodologia utilizada atualmente, com o uso de armação de $2 \mathrm{~m}^{2}$ pode resultar em valores de perdas menores que as perdas reais, se considerarmos o alto $\mathrm{CV}$ encontrado para esta armação (Tabela 1), com os valores de perdas da armação de $3 \mathrm{~m}^{2}$ mais próximos dos valores reais.

Pode-se afirmar ainda que o alto valor de CV encontrado para as perdas com armação de $2 \mathrm{~m}^{2}$ prejudica as análises, pois no caso desta pesquisa, a perda indicada pela armação de $2 \mathrm{~m}^{2}$ está abaixo do valor tolerável, enquanto para a armação de $3 \mathrm{~m}^{2}$ este valor ficou acima do valor tolerável, que é de $60 \mathrm{~kg} \mathrm{ha}^{-1}$.

Observa-se também que não houve interação entre todos os fatores, indicando que um fator não influencia o outro em relação a perdas totais na colheita da cultura da soja.

A quantificação das perdas totais na colheita da soja com armação de $2 \mathrm{~m}^{2}$, apresentou coeficiente de variação maior que o da armação de $3 \mathrm{~m}^{2}$.

A velocidade de deslocamento e a abertura entre o cilindro e o côncavo não afetaram as perdas totais na colheita de soja.

A armação de $3 \mathrm{~m}^{2}$ quantificou valores de perdas totais maiores do que a armação de $2 \mathrm{~m}^{2}$.

\section{REFERÊNCIAS BIBLIOGRÁFICAS}

ANDRIOLI, I.; CENTURION, J. F. Levantamento detalhado dos solos da Faculdade de Ciências Agrárias e Veterinárias de Jaboticabal. In: CONGRESSO BRASILEIRO DE CIÊNCIA DO SOLO, 27., 1999, Brasília, DF. Anais... Brasília, DF: Sociedade Brasileira de Ciência do Solo, 1999. CD-ROM.

CAMPOS, M. A. O.; SILVA, R. P.; CARVALHO FILHO, A.; MESQUITA, H. C. B.; ZABANI, S. Perdas na colheita mecanizada de soja no estado de Minas Gerais. Engenharia Agrícola, Jaboticabal, v. 25, n. 1, p. 207-213, jan./abr. 2005.

CARVALHO FILHO, A. C.; CORTEZ, J. W.; SILVA, R. P.; ZAGO, M. S. Perdas na colheita mecanizada da soja no triângulo mineiro. In: CONGRESSO DE INICIAÇÃO CIENTÍFICA E DE PESQUISADORES DA FUNDAÇÃO EDUCACIONAL DE ITUVERAVA, 1., 2004, Ituverava. Anais... Ituverava: Fundação Educacional de Ituverava, 2004. CD-ROM. 
COSTA, N. P.; MESQUITA, C. M.; MAURINA, A. C.; FRANÇA-NETO, J. B.; PEREIRA, J. E.; KRZYZANWSKI, F. C.; HENNING, A. A. Avaliação da qualidade de sementes e grãos de soja provenientes da colheita mecanizada, em diferentes regiões do Brasil. Engenharia Agrícola, Jaboticabal, v. 22, n. 2, p. 211-19, 2002.

COSTA, N. P.; TAVARES, L. C. V. Fatores responsáveis pelos elevados percentuais de perdas de grãos durante a colheita mecânica em soja. Informativo ABRATES, [S.l.], v. 5, n. 1, abr. 1995.

EMPRESA BRASILEIRA DE PESQUISA AGROPECUÁRIA. Centro nacional de pesquisas de solos. Sistema brasileiro de classificação de solos. Brasília, DF, 1999. $412 \mathrm{p}$.

EMPRESA BRASILEIRA DE PESQUISA AGROPECUÁRIA. Tecnologias de produção da soja no Paraná 2004. Londrina: Embrapa Soja, 2003. 218 p.

HAMER, E.; PESKE, S. T. Colheita de sementes de soja com alto grau de umidade: I. qualidade física. Revista Brasileira de Sementes, Brasília, v. 19, n. 1, p. 106-110, 1997.

KUNZ, V. L.; FEY, E.; PRIMO, M. A.; GURGACZ, F. Perdas de grãos livres e grãos nas vagens na colheita de soja utilizando colhedoras autopropelidas. In: CONGRESSO BRASILEIRO DE ENGENHARIA AGRÍCOLA, 34., 2005, Canoas, RS. Anais... Jaboticabal: Sociedade Brasileira de Engenharia Agrícola, 2005. CD-ROM.

MESQUITA, C. de M.; COSTA, N. P.; MANTOVANI, E. C.; ANDRADE, J. G. M.; FRANÇA NETO, J. B.; SILVA, J. G.; FONSECA, J. R.; PORTUGAL, F. A. F.; GUIMARÃES SOBRINHO, J. B. Manual do produtor: como evitar desperdícios nas colheitas da soja, do milho e do arroz. Londrina: Embrapa-CNPS ${ }_{0}$ 1998. p. 19-22.

MESQUITA, C. de M.; COSTA, N. P.; PEREIRA, J. E.; MAURINA, A. C.; ANDRADE, J. G. M. Colheita mecânica da soja: avaliação das perdas e da qualidade física do grão. Engenharia Agrícola, Jaboticabal, v. 18, n. 3, p. 44-53, mar. 1999.
MESQUITA, C. de M.; COSTA, N. P.; PEREIRA, J. E.; MAURINA, A. C.; ANDRADE, J. G. M. Caracterização da colheita mecanizada da soja no Paraná. Engenharia Agrícola, Jaboticabal, v. 21, n. 2, p. 197-205, maio 2001.

MESQUITA, C. de M.; COSTA, N. P.; PEREIRA, J. E.; MAURINA, A. C.; ANDRADE, J. G. M. Perfil da colheita mecânica da soja no Brasil: safra 1998/1999. Engenharia Agrícola, Jaboticabal, v. 22, n. 3, p. 398-406, set. 2002.

MESQUITA, C. de M.; GAUDENCIO, C. A. Medidor de perdas na colheita de soja e trigo. Londrina: Embrapa$\mathrm{CNPS}_{\mathrm{O}}, 1982.8$ p. (Comunicado técnico, 15).

MORAES, M. L. B.; REIS, Â. V.; TOESCHER, C. F.; MACHADO, A. L. T. Máquinas para colheita e processamento dos grãos. Pelotas: UFPel, 1996.

PINHEIRO NETO, R.; GAMERO, C. A. Efeito da colheita mecanizada nas perdas quantitativas de grãos de soja (Glycine max (L.) Merrill). Energia na Agricultura, Botucatu, v. 14, n. 1, p. 69-81, 1999.

PORTELlA, J. A. Colheita de grãos mecanizada: implementos, manutenção e regulagem. Viçosa: Aprenda Fácil, 2000a. 190 p.

PORTELLA, J. A. Perdas quali-quantitativas de trigo em diferentes épocas de colheita. In: CONGRESSO BRASILEIRO DE ENGENHARIA AGRÍCOLA, 29., 2000, Fortaleza. Anais... Jaboticabal: Sociedade Brasileira de Engenharia Agrícola, 2000b. CD-ROM.

SILVA, R. P.; CAIXETA, R. V.; SILVA, E. C. Perdas de grãos ocorridas na pré-colheita e mecanismos internos de uma colhedora de milho (Zea mays). In: CONGRESSO BRASILEIRO DE ENGENHARIA AGRÍCOLA, 27., 1998, Poços de Caldas. Anais... Lavras: UFLA/SBEA, 1998. v. 3, p. 214-216.

SILVA, S. G.; CORONEL, C. E. I.; RAMÍREZ, M. A.; VELASCO, P. E. Análisis de la calidad del grano durante la cosecha de cebada con la combinada John Deere 9500.

Revista Ciências Técnicas Agropecuárias, [S.1.], v. 13, n. 1, p. 25-29, 2004. 